\title{
Anisotropie des transitions interbandes dans le nitrure de bore hexagonal
}

\author{
R. Mamy $\left({ }^{*}\right)$, J. Thomas $\left({ }^{* *}\right)$, G. Jezequel $\left({ }^{* *}\right)$ et J. C. Lemonnier $\left({ }^{* *}\right)$ \\ L.U.R.E., Bâtiment 209C, Université de Paris-Sud, 91405 Orsay Cedex, France

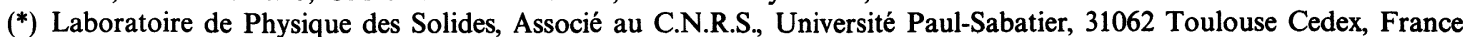 \\ (**) Laboratoire de Spectroscopie, Equipe Associée au C.N.R.S., Université de Rennes, Avenue du Général-Leclerc, 35041 \\ Rennes Cedex, France
}

(Reçu le 22 juin 1981, accepté le 29 septembre 1981)

\begin{abstract}
Résumé. - Les fonctions diélectriques ordinaires et extraordinaires du nitrure de bore hexagonal ont été calculées à partir de mesures multi-angle du pouvoir réflecteur pour une énergie de rayonnement de $5 \mathrm{eV}$ à $30 \mathrm{eV}$. Les spectres obtenus sont discutés en termes de transitions interbandes.
\end{abstract}

\begin{abstract}
The ordinary and extraordinary dielectric functions of hexagonal boron nitride were calculated after measuring the reflecting power with a multiangle method. The incident energy radiation extended from $5 \mathrm{eV}$ to $30 \mathrm{eV}$. The spectra are discussed as interband transitions.
\end{abstract}

Le nitrure de bore (BN) hexagonal est un composé lamellaire très anisotrope de structure cristallographique voisine de celle du graphite ; alors que ce dernier est un semi-métal, le BN est isolant. Les mesures optiques publiées n'ont été faites qu'autour du gap, ainsi les dernières mesures d'absorption de couches minces donnent pour le gap optique une valeur de l'ordre de 5,5 eV [1]. Il était intéressant d'étendre les mesures jusqu'à l'ultraviolet extrême pour étudier les singularités bidimensionnelles et aussi pour calculer l'anisotropie des fonctions optiques. Pour le BN cette anisotropie n'est connue que par les mesures de pertes d'énergie d'électrons [2]. Par contre, dans le cas du graphite, elle a été calculée et interprétée à partir des résultats de pertes d'énergie [3], ainsi qu'à partir des mesures optiques [4].

La structure de bandes du BN hexagonal est connue $[1,5,6]$; les bandes de valence supérieures, la bande $\pi\left(\mathrm{p}_{z}\right)$ d'une part et les bandes $\sigma\left(\mathrm{s}, \mathrm{p}_{x}, \mathrm{p}_{y}\right.$ en $\left.\Gamma\right)$ d'autre part, donnent deux maximums de densité d'états bien séparés; les états $2 \mathrm{~s}$ de l'azote, situés à $13 \mathrm{eV}$ au-dessous de la bande $\pi$, contribuent aux transitions dans le domaine spectral étudié. Un modèle bidimensionnel peut servir de première approche (Fig. 1). Dans un modèle tridimensionnel, seules les bandes $\pi$ sont affectées de façon sensible par la levée de dégénérescence.

1. Méthode de mesure. - A partir d'une lumière partiellement polarisée linéairement, on mesure le pouvoir réflecteur sous diverses incidences (de $20^{\circ}$ à

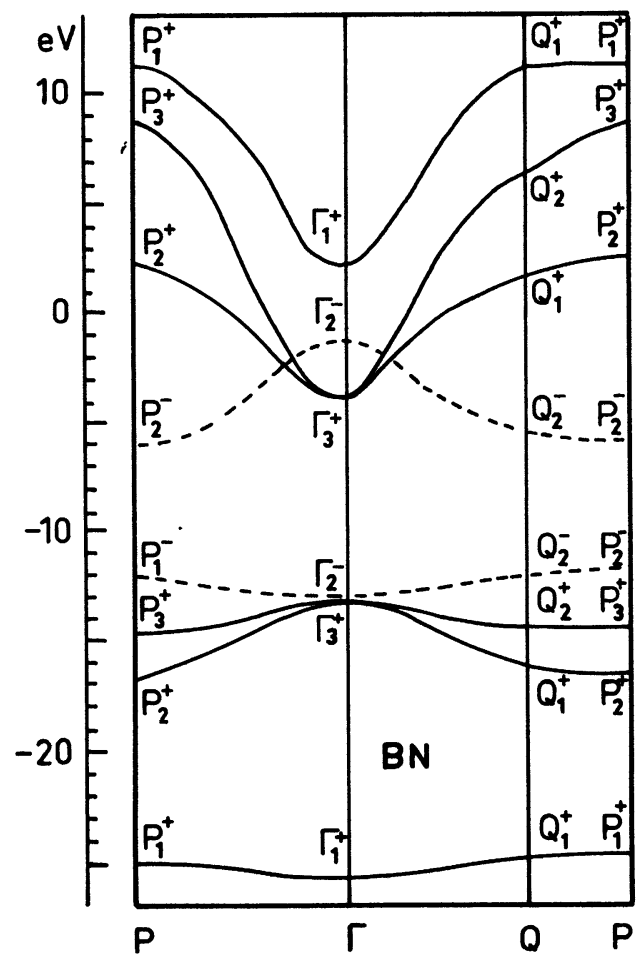

Fig. 1. - Schéma de bandes bidimensionnel de BN d'après [5]. Les bandes $\pi$ sont tracées en pointillés.

[Two-dimensional band scheme of BN (from reference [5]). The dotted lines correspond to the $\pi$-bands.]

$60^{\circ}$, tous les $5^{\circ}$ ) et deux positions du plan d'incidence par rapport à l'ellipse de polarisation. Connaissant le 
taux de polarisation de la lumière, on calcule les pouvoirs réflecteurs $R_{\mathrm{s}}\left(\theta, n_{\mathrm{o}}, k_{\mathrm{o}}\right)$ et $R_{\mathrm{p}}\left(\theta, n_{\mathrm{o}}, k_{\mathrm{o}}, n_{\mathrm{e}}, k_{\mathrm{e}}\right)$ [7] correspondant à une excitation purement s et purement $\mathrm{p}$ (les indices o et e correspondant aux fonctions optiques ordinaires et extraordinaires). Un procédé d'ajustage basé sur les moindres carrés [8] appliqués à $R_{\mathrm{s}}$ fournit $\left(n_{\mathrm{o}}, k_{\mathrm{o}}\right)$; puis connaissant les fonctions ordinaires, on applique le même procédé sur $R_{\mathrm{p}}\left(\theta, n_{\mathrm{o}}\right.$, $\left.k_{\mathrm{e}}, n_{\mathrm{e}}, k_{\mathrm{e}}\right)$ ce qui nous donne $\left(n_{\mathrm{e}}, k_{\mathrm{e}}\right)$. Les mesures ont été effectuées au L.U.R.E. (Orsay) à l'aide du rayonnement synchrotron entre 5 et $30 \mathrm{eV}$, avec un taux de polarisation de la lumière compris entre 0,4 et 0,8 . L'échantillon étudié est constitué de microcristaux dont les axes optiques sont parallèles (nitrure de bore pyrolytique).

2. Résultats. - Nous présentons (Figs. 2 et 3 ) les fonctions diélectriques $\varepsilon_{2}$ ordinaires et extraordinaires, de 5 à $9 \mathrm{eV}$ et $13 \mathrm{à} 30 \mathrm{eV}$, la région intermédiaire ne présentant pas de structures notables; les fonctions - $\operatorname{Im}(1 / \varepsilon)$ correspondantes sont données figures 4 et 5 ; nous interpréterons les structures observées sur les

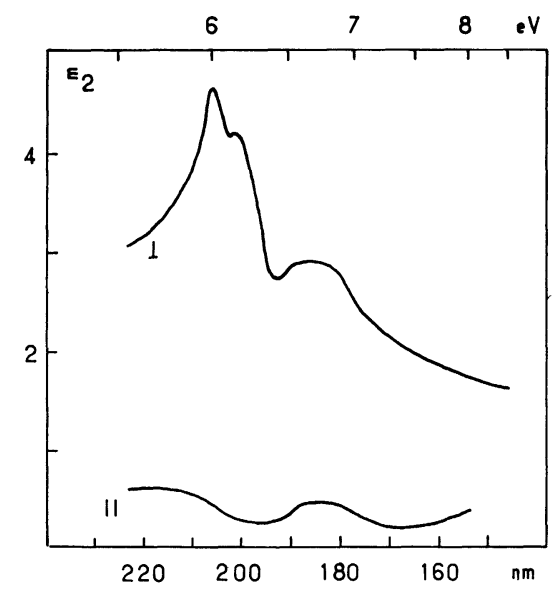

Fig. 2. - Fonctions diélectriques $\varepsilon_{2 \perp}$ et $\varepsilon_{2 \|}$ de $5 \mathrm{eV}$ à $9 \mathrm{eV}$. [Dielectric functions $\varepsilon_{2 \perp}$ and $\varepsilon_{2 \|}$ from $5 \mathrm{eV}$ to $9 \mathrm{eV}$.]

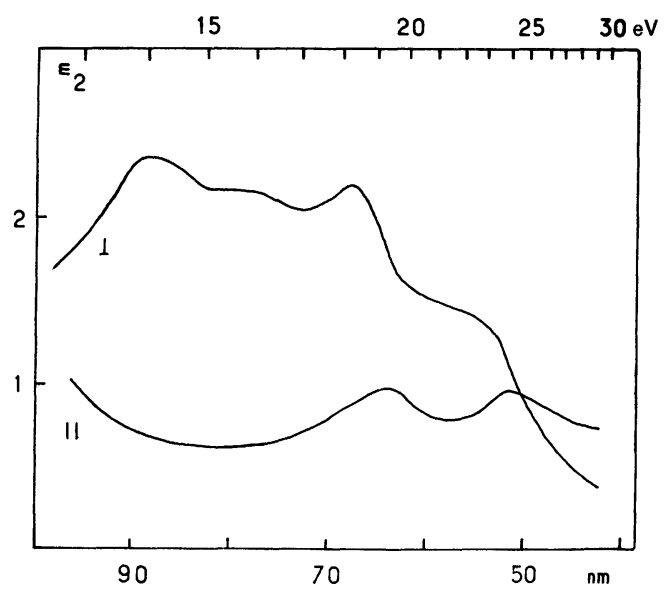

Fig. 3. - Fonctions diélectriques $\varepsilon_{2 \perp}$ et $\varepsilon_{2 \|}$ de $13 \mathrm{eV}$ à $30 \mathrm{eV}$. [Dielectric functions $\varepsilon_{2 \perp}$ and $\varepsilon_{2 \|}$ from $13 \mathrm{eV}$ to $30 \mathrm{eV}$.]

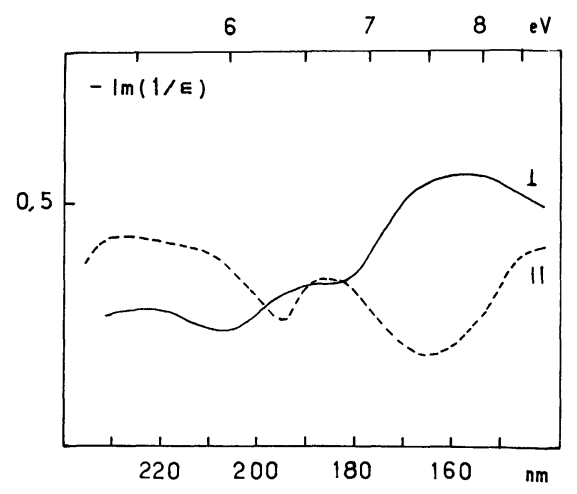

Fig. 4. - Fonctions diélectriques $-\operatorname{Im}\left(1 / \varepsilon_{\perp}\right)$ et $-\operatorname{Im}\left(1 / \varepsilon_{\|}\right)$de $5 \mathrm{eV}$ à $9 \mathrm{eV}$.

[Dielectric functions $-\operatorname{Im}\left(1 / \varepsilon_{\perp}\right)$ and $-\operatorname{Im}\left(1 / \varepsilon_{\|}\right)$from $5 \mathrm{eV}$ to $9 \mathrm{eV}$.]

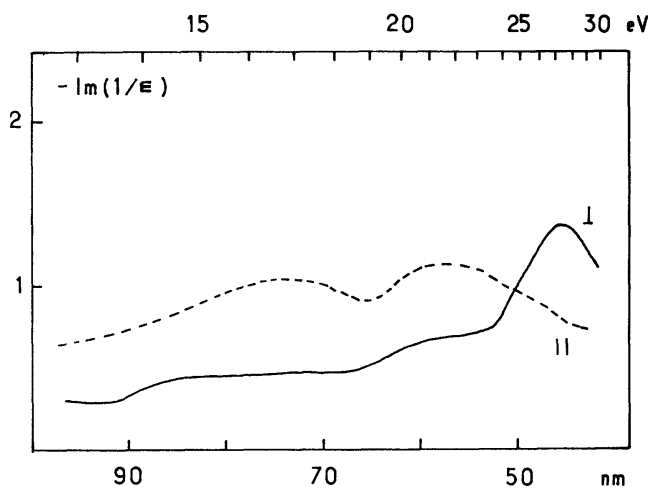

Fig. 5. - Fonctions diélectriques $-\operatorname{Im}\left(1 / \varepsilon_{\perp}\right)$ et $-\operatorname{Im}\left(1 / \varepsilon_{\|}\right)$de $13 \mathrm{eV}$ à $30 \mathrm{eV}$.

[Dielectric functions $-\operatorname{Im}\left(1 / \varepsilon_{\perp}\right)$ and $-\operatorname{Im}\left(1 / \varepsilon_{\|}\right)$from $13 \mathrm{eV}$ to $30 \mathrm{eV}$.]

spectres en termes de transitions interbandes, principalement entre points $Q$ de la zone de Brillouin, car ces dernières donnent de fortes singularités dans la densité d'états composée [5].

La fonction $\varepsilon_{2 \perp}$ est caractérisée à basse énergie (Fig. 2) par deux pics à $6 \mathrm{eV}$ et $6,7 \mathrm{eV}$. La densité d'états composée a été calculée [5] pour les transitions $\pi \rightarrow \pi$ en fonction des règles de sélection : il apparaît, pour $E \perp c,(E$ champ électrique du rayonnement, $c$ axe optique du cristal), un seul pic vers $6,2 \mathrm{eV}$ dans le cas bidimensionnel (singularité bidimensionnelle type $M_{1}$ due à la transition $\mathrm{Q}_{2}^{-} \rightarrow \mathrm{Q}_{2}^{-}$) tandis que dans le cas tridimensionnel, deux pics apparaissent à $6,2 \mathrm{eV}$ et $7 \mathrm{eV}$; ainsi, nous pouvons attribuer, sur le spectre de la fonction $\varepsilon_{2 \perp}$ (Fig. 2), le pic à $6 \mathrm{eV}$ à la transition $\mathrm{Q}_{2 \mathrm{~g}}^{-} \rightarrow \mathrm{Q}_{2 \mathrm{u}}^{-}$et celui à 6,7 eV à $\mathrm{Q}_{2 \mathrm{u}}^{-} \rightarrow \mathrm{Q}_{2 \mathrm{~g}}^{-}$. Le dédoublement du premier pic de $\varepsilon_{2 \perp}$ peut s'expliquer par la contribution de la transition $\mathrm{K}_{3}^{-} \rightarrow \mathrm{K}_{3}^{-}$[5]. Pour $E / / c$, les transitions ne sont permises que dans un modèle tridimensionnel, les calculs donnent un seul pic vers 6,6 eV. La fonction $\varepsilon_{2 \|}$ (Fig. 2) a des valeurs faibles et la position des structures est en désaccord 
avec celle prévue par les calculs; le deuxième pic est à la même énergie que précédemment tandis que le premier est fortement atténué et déplacé vers les basses énergies. Comme nous ne trouvons d'accord quantitatif avec aucun modèle, dans le cas $E / / c$, il faut en conclure que les bandes $\pi$ sont différentes de celles calculées ou qu'il doit y avoir une plus grande contribution des transitions en $\Gamma$, ce qui favoriserait plus le schéma de bandes [6].

La fonction $\varepsilon_{2 \perp}$ présente à plus haute énergie (Fig. 3) des structures attribuables à des transitions $\sigma \rightarrow \sigma: \mathrm{Q}_{2}^{+} \rightarrow \mathrm{Q}_{1}^{+}$à $14 \mathrm{eV}, \mathrm{Q}_{1}^{+} \rightarrow \mathrm{Q}_{1}^{+}$à $18,5 \mathrm{eV}$ et $2 \mathrm{~s}_{\mathrm{N}} \rightarrow \mathrm{Q}_{1}^{+}$à $23 \mathrm{eV}$ en s'appuyant sur le schéma de bandes [5] (Fig. 1). Il faut noter que les points $\mathrm{Q}_{2}^{-}, \mathrm{Q}_{2}^{+}$, $\mathrm{Q}_{1}^{+}$des bandes de valence sont à l'origine des fortes densités d'états de valence [6]. La fonction $\varepsilon_{2 \|}$ dans le même domaine d'énergie est caractérisée par un transfert vers les hautes énergies de la force d'oscillateur due à l'absence de transition $\sigma \rightarrow \pi$ entre $11 \mathrm{eV}$ et $18 \mathrm{eV}$; les structures s'interprètent par des transitions $2 \mathrm{~s}_{\mathrm{N}} \rightarrow \mathrm{Q}_{2}^{-}$à $19,5 \mathrm{eV}$ et $2 \mathrm{~s}_{\mathrm{N}} \rightarrow \Gamma_{2}^{-}$à $24 \mathrm{eV}$.

Les calculs de $-\operatorname{Im}(1 / \varepsilon)$ (Figs. 4 et 5$)$ permettent d'établir une comparaison avec les mesures d'anisotropie de pertes d'énergie d'électrons faites sur BN [2]. La fonction - $\operatorname{Im}\left(1 / \varepsilon_{\perp}\right)$ présente deux maximums à $7,75 \mathrm{eV}$ et $27 \mathrm{eV}$ qui correspondent respectivement à l'excitation collective des électrons $\pi$ et $\pi+\sigma(8,8 \mathrm{eV}$ et $25,5 \mathrm{eV}$ expérimentalement [2]). Dans le cas de - $\operatorname{Im}\left(1 / \varepsilon_{\|}\right)$à basse énergie, les deux structures à 5,5 et
$6,7 \mathrm{eV}$ correspondent au pic à $6 \mathrm{eV}$ des résultats de pertes d'énergie d'électrons.

E. Tosatti et F. Bassani [5] ont montré que dans le cas d'un cristal uniaxe les modes propres ne sont en général ni transverses ni longitudinaux et mélangent vibration lumineuse et oscillation de plasma et que pour une direction quelconque du vecteur $k$ de l'oscillation collective, la condition $\left(\varepsilon_{\perp} k_{\perp}^{2}+\varepsilon_{\|} k_{\|}^{2}\right) / k^{2}=0$ donne les maximums de la fonction perte d'énergie. Dans le cas où le vecteur $k$ est presque parallèle à l'axe optique de l'échantillon, on distingue deux types de maximums de la fonction perte d'énergie, les uns ont le caractère d'une oscillation collective, les autres sont des transitions interbandes. Certaine excitation appelée anisotron, collective dans le cas $k \perp c$, devient une transition interbande dans le cas $k / / c$; c'est ainsi que dans nos résultats le plasmon des électrons $\pi$ disparaît dans le cas d'une excitation parallèle à l'axe optique, pour donner des structures correspondant à des transitions interbandes, c'est ce que l'on constate sur les structures à $5,5 \mathrm{eV}$ et $6,7 \mathrm{eV}$ de la fonction - Im $\left(1 / \varepsilon_{\|}\right)$qui correspondent aux structures que l'on observe sur $\varepsilon_{2 \perp}$ à la même énergie, cela confirme la théorie des auteurs précédents.

Remerciements. - Nous tenons à remercier pour leur aide le personnel du Laboratoire de l'Accélérateur Linéaire et en particulier le groupe « Anneaux ».

\section{Bibliographie}

[1] Zunger, A., Katzir, A., Halperin, A., Phys. Rev. B 13 (1976) 5560.

[2] Cazaux, J., C.R. Hebd. Séan. Acad. Sci. Paris 270 (1970) 700.

[3] Tosatti, E., Bassani, F., Nuovo Cimento X 65B (1970) 161.

[4] Klucker, R., Skibowski, M., Steinmann, W., Phys. Status Solidi (b) 65 (1974) 703.
[5] Doni, E., Pastori Parravicini, G., Nuovo Cimento LXIV B 1 (1969) 117

[6] Zupan, J., Phys. Rev. B 6 (1972) 2477.

[7] Uzan, E., Thèse, Paris (1971) et Optica Acta 15, 3 (1968) 237.

[8] ThOMAs, J. et al., Optica Acta 19, 12 (1972) 983. 